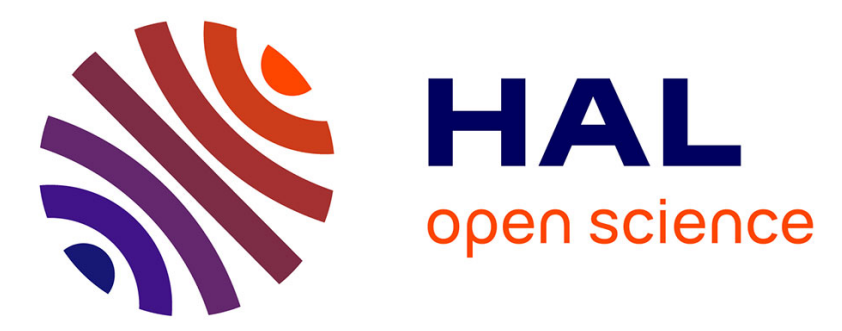

\title{
Infrared magneto-spectroscopy of two- dimensional and three-dimensional massless fermions: A comparison
}

M. Orlita, C. Faugeras, A.-L. Barra, G. Martinez, M. Potemski, D M Basko, M. Zholudev, F. Teppe, W. Knap, V. Gavrilenko, et al.

\section{- To cite this version:}

M. Orlita, C. Faugeras, A.-L. Barra, G. Martinez, M. Potemski, et al.. Infrared magneto-spectroscopy of two- dimensional and three-dimensional massless fermions: A comparison. Journal of Applied Physics, 2015, 117 (11), pp.112803. 10.1063/1.4913828 . hal-01784078

\section{HAL Id: hal-01784078 \\ https://hal.science/hal-01784078}

Submitted on 3 May 2018

HAL is a multi-disciplinary open access archive for the deposit and dissemination of scientific research documents, whether they are published or not. The documents may come from teaching and research institutions in France or abroad, or from public or private research centers.
L'archive ouverte pluridisciplinaire HAL, est destinée au dépôt et à la diffusion de documents scientifiques de niveau recherche, publiés ou non, émanant des établissements d'enseignement et de recherche français ou étrangers, des laboratoires publics ou privés. 


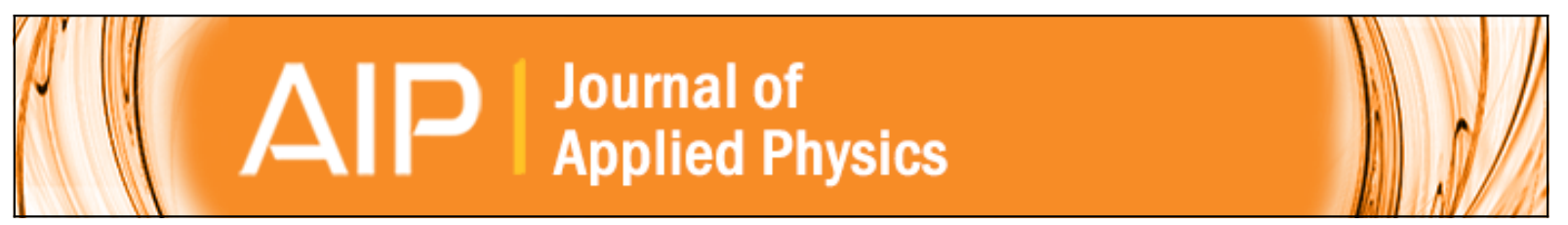

Infrared magneto-spectroscopy of two-dimensional and three-dimensional massless fermions: A comparison

M. Orlita, C. Faugeras, A.-L. Barra, G. Martinez, M. Potemski, D. M. Basko, M. S. Zholudev, F. Teppe, W. Knap, V. I. Gavrilenko, N. N. Mikhailov, S. A. Dvoretskii, P. Neugebauer, C. Berger, and W. A. de Heer

Citation: Journal of Applied Physics 117, 112803 (2015); doi: 10.1063/1.4913828

View online: http://dx.doi.org/10.1063/1.4913828

View Table of Contents: http://scitation.aip.org/content/aip/journal/jap/117/11 ?ver=pdfcov

Published by the AIP Publishing

\section{Articles you may be interested in}

Nernst and Seebeck effects in $\mathrm{HgTe} / \mathrm{CdTe}$ topological insulator

J. Appl. Phys. 117, 124301 (2015); 10.1063/1.4915933

Terahertz magneto-optical spectroscopy of a two-dimensional hole gas

Appl. Phys. Lett. 106, 031902 (2015); 10.1063/1.4906282

Two-dimensional semimetal in HgTe-based quantum wells

Low Temp. Phys. 37, 202 (2011); 10.1063/1.3573648

Realization of photoreflectance spectroscopy in very-long wave infrared of up to $20 \mu \mathrm{m}$ Appl. Phys. Lett. 95, 041908 (2009); 10.1063/1.3193546

Study of impurity states in p-type $\mathrm{Hg} 1-\mathrm{x} \mathrm{Cd} x \mathrm{Te}$ using far-infrared spectroscopy

Appl. Phys. Lett. 73, 1538 (1998); 10.1063/1.122198

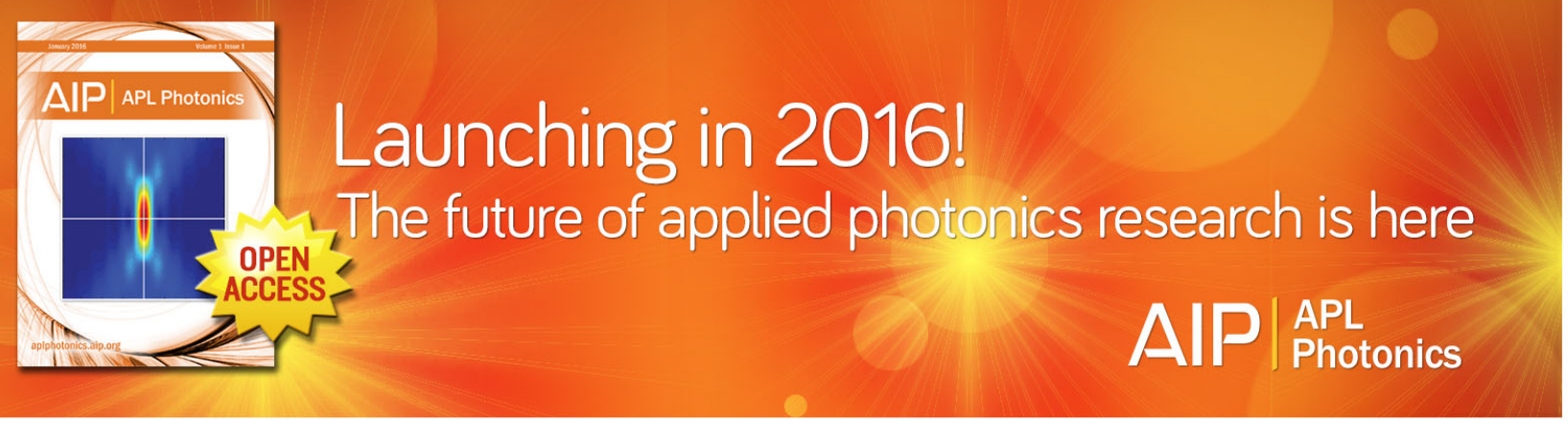




\title{
Infrared magneto-spectroscopy of two-dimensional and three-dimensional massless fermions: A comparison
}

\author{
M. Orlita,,${ }^{1,2, a)}$ C. Faugeras, ${ }^{1}$ A.-L. Barra, ${ }^{1}$ G. Martinez, ${ }^{1}$ M. Potemski, ${ }^{1}$ D. M. Basko, ${ }^{3}$ \\ M. S. Zholudev, ${ }^{4,5}$ F. Teppe, ${ }^{4}$ W. Knap, ${ }^{4}$ V. I. Gavrilenko, ${ }^{5}$ N. N. Mikhailov, ${ }^{6}$ S. A. Dvoretskii, ${ }^{6}$ \\ P. Neugebauer, ${ }^{7}$ C. Berger, ${ }^{8,9}$ and W. A. de $\mathrm{Heer}^{8}$ \\ ${ }^{1}$ Laboratoire National des Champs Magnétiques Intenses, CNRS-UJF-UPS-INSA, 38042 Grenoble, France \\ ${ }^{2}$ Faculty of Mathematics and Physics, Charles University, Ke Karlovu 5, 121 16 Prague 2, Czech Republic \\ ${ }^{3}$ LPMMC UMR 5493, Université Grenoble 1/CNRS, B.P. 166, 38042 Grenoble, France \\ ${ }^{4}$ Laboratoire Charles Coulomb (L2C), UMR CNRS 5221, GIS-TERALAB, Université Montpellier II, \\ 34095 Montpellier, France \\ ${ }^{5}$ Institute for Physics of Microstructures, RAS, Nizhny Novgorod GSP-105 603950, Russia \\ ${ }^{6}$ A.V. Rzhanov Institute of Semiconductor Physics, Siberian Branch, Russian Academy of Sciences, \\ Novosibirsk 630090, Russia \\ ${ }^{7}$ Institut für Physikalische Chemie, Universität Stuttgart, Pfaffenwaldring 55, 70569 Stuttgart, Germany \\ ${ }^{8}$ School of Physics, Georgia Institute of Technology, Atlanta, Georgia 30332, USA \\ ${ }^{9}$ Institut Néel/CNRS-UJF BP 166, F-38042 Grenoble Cedex 9, France
}

(Received 5 September 2014; accepted 14 September 2014; published online 16 March 2015)

\begin{abstract}
Here, we report on a magneto-optical study of two distinct systems hosting massless fermionstwo-dimensional graphene and three-dimensional $\mathrm{HgCdTe}$ tuned to the zero band gap condition at the point of the semiconductor-to-semimetal topological transition. Both materials exhibit, in the quantum regime, a fairly rich magneto-optical response, which is composed from a series of intraand interband inter-Landau level resonances with for massless fermions typical $\sqrt{B}$ dependence. The impact of the system's dimensionality and of the strength of the spin-orbit interaction on the optical response is also discussed. (C) 2015 AIP Publishing LLC.
\end{abstract}

[http://dx.doi.org/10.1063/1.4913828]

\section{INTRODUCTION}

Solid-state physics and quantum electrodynamics, with its relativistic (massless) particles, meet in steadily expanding class of materials. Those include, 1D carbon nanotubes, ${ }^{1}$ 2D graphene, ${ }^{2,3}$ topological-insulator surfaces, ${ }^{4-8}$ semiconductors with a giant Rashba-type spin splitting, ${ }^{9}$ and most recently, the systems with $3 \mathrm{D}$ conical dispersion-with Dirac $^{10-14}$ or Kane ${ }^{15}$ fermions.

Within recent past years, optical spectroscopy, often in the infrared spectral region, appeared to be an efficient experimental tool to study these appealing Dirac-type materials, in particular, when this technique is combined with the application of magnetic fields. ${ }^{14-27}$ The magnetic field significantly changes the character of the motion of charge carriers (cyclotron motion) and induces a considerable modulation of the density of electronic states due to the appearance of Landau levels (LLs). The Dirac-type systems, with conical bands and therefore strongly non-equidistant Landau levels, give rise to a fairly rich magneto-optical response, involving both intra- (cyclotron resonance) and inter-band excitations with a characteristic $\sqrt{B}$ dependence on the applied magnetic field. ${ }^{16-19}$

In this paper, we compare the magneto-optical response of two specific Dirac materials-multilayer epitaxial graphene (MEG) and bulk mercury cadmium telluride (MCT) tuned, with the cadmium concentration, to a nearly zeroband gap condition. Both these systems display well-defined

${ }^{a)}$ Electronic address: milan.orlita@lncmi.cnrs.fr spin-degenerate conical bands. In graphene, the 2D cones are located in the corners of the Brillouin zone; a gapless MCT displays a single cone at the $\Gamma$ point, which is additionally crossed by a flat (heavy hole) hole band at the cones' vertex. The schematic band structure has been plotted for both systems in Fig. 1.

\section{EXPERIMENT}

The studied samples are standard multilayer epitaxial graphene specimens ${ }^{28}$ grown on the C-terminated surface of silicon carbide $(4 \mathrm{H}-\mathrm{SiC}[000 \overline{1}])$ with intentional thickness of 50 layers. A significant part of layers displays an electronic band structure identical of an isolated graphene monolayer.
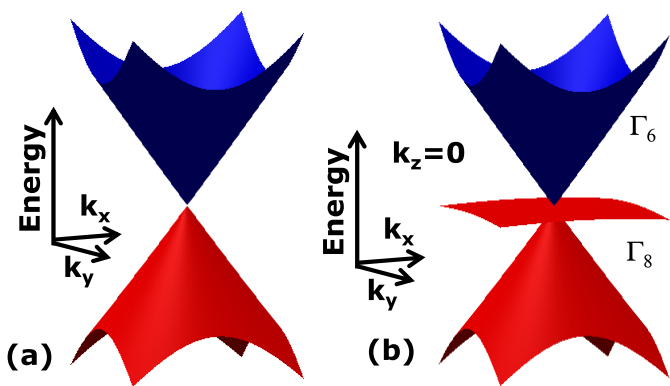

FIG. 1. Schematic band structures of graphene (a) and of MCT with a zero energy band gap (b). While the band structure of graphene contains two 2D Dirac cones (around $K$ and $K^{\prime}$ points of the Brillouin zone), a single 3D conical band appears in the gapless MCT (at the $\Gamma$ point), which is additionally crossed by rather flat (heavy hole) band. In both parts, blue colour corresponds to the conduction band and the valence bands are depicted in red. 
This results from the characteristic rotational stacking of these graphene sheets, ${ }^{29}$ and it is confirmed by micro-Raman spectra measured on our sample, which show a singlecomponent $2 \mathrm{D}$ band $^{30,31}$ (with some Bernal-stacked residuals on selected locations). The sheets studied in this experiment are quasi-neutral; ${ }^{20}$ only several layers close to the interface and on the surface of MEG become significantly doped (up to $\left.10^{13} \mathrm{~cm}^{-2}\right) .^{32}$

The MCT sample was grown using standard molecularbeam epitaxy on a (013)-oriented semi-insulating GaAs substrate. The growth sequence started with ZnTe and CdTe buffer layers, followed by the MCT epilayer with gradually changing cadmium content $x$, see Ref. 15 for details. The 3D conical band develops at the point of semiconductor-to-semimetal transition (around $x \approx 0.17$ at low temperatures). The investigated specimen contains a relatively thin layer $(d \cong 3.2 \mu \mathrm{m})$ with this particular Cd concentration, which is suitable for the transmission spectroscopy. The sample exhibits a weak $n$-type background doping.

To measure the infrared transmittance, the sample was exposed to the radiation of a globar, which was analyzed by a Fourier transform spectrometer and delivered to the sample via light-pipe optics. The transmitted light was detected by a composite bolometer which was operated at $T=1.8 \mathrm{~K}$ and which was placed directly below the sample. Measurements were done in the Faraday configuration, using a superconducting or resistive magnet.

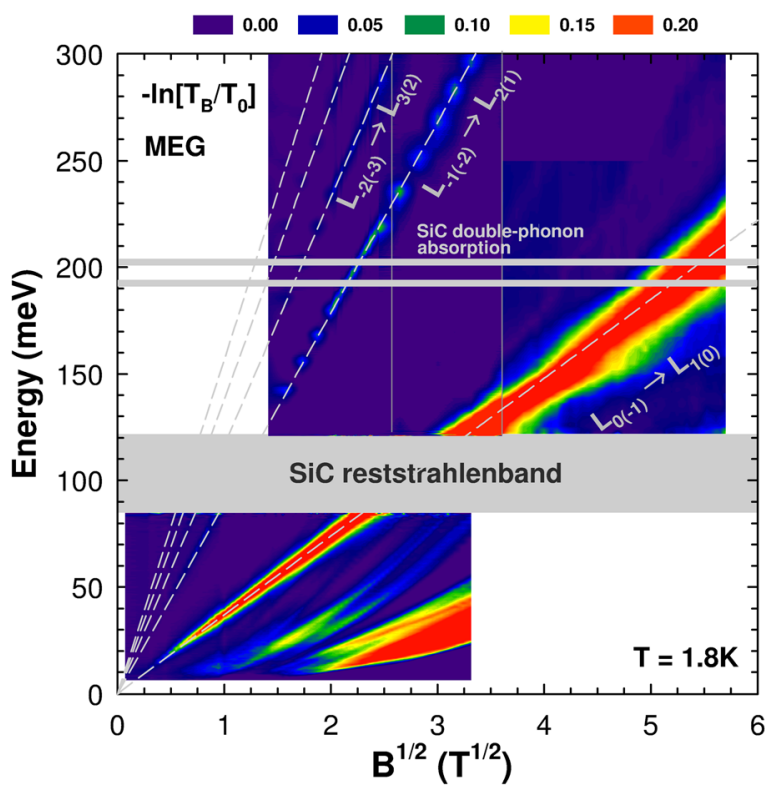

FIG. 2. Relative change of MEG absorbance with the applied magnetic field $\left(A_{B} / A_{0}\right)$ plotted as a false colour map. The data show a series of inter-LL excitations in graphene with a perfect $\sqrt{B}$ dependence, the low-energy/low$B$ response is dominated by absorption in highly doped graphene layers, a-few layer graphene stacks (e.g., bilayer graphene inclusions ${ }^{33}$ ) and also bulk graphite inclusions. Interaction with the $K$ point phonons in graphene are responsible for a small kink visible in the evolution of $\mathrm{L}_{-1(0)} \rightarrow \mathrm{L}_{0(1)}$ transition around $B \approx 20 \mathrm{~T}$ (at photon energy of $\approx 160 \mathrm{meV}$ ). ${ }^{25}$ The data have been collected on several MEG samples with very similar properties, but with a slightly different number of layers. Therefore, the strength of absorbance differs in four separated windows. The grey areas correspond to region with strong absorption on phonons in the $\mathrm{SiC}$ substrate.

\section{RESULTS AND DISCUSSION}

The magneto-absorbance of the MEG and of the gapless MCT specimens is plotted in Figs. 2 and 3, respectively, in a form of false colour maps. In both cases, the observed magneto-optical response provides a clear signature of massless particles: the observed spectrum is composed of a series of inter-LL resonances, which follow a linear in $\sqrt{B}$ dependence in a broad range of magnetic fields. In the quasi-neutral sheets of MEG specimens, such a dependence may be traced over more than three orders of magnitude, down to the millitesla range. ${ }^{20,25}$

To identify the individual absorption lines, we briefly describe the LL spectrum of each investigated material. In graphene, which is a strictly $2 \mathrm{D}$ material, the electronic spectrum in a magnetic field consists of discrete LLs, which are four times degenerate due to the valley and spin degrees of freedom $(n=0, \pm 1, \pm 2 \ldots)$

$$
E_{n}=\operatorname{sign}(n) v_{F}^{\mathrm{G}} \sqrt{2 e \hbar B|n|} .
$$

In the bulk gapless MCT, Landau levels (or Landau bands due to their dispersive character) display a more complex form, with the band and LL index, $\zeta=0, \pm 1$ and $n=0$, $1,2 \ldots$, respectively,

$$
E_{\zeta, n, \sigma}\left(k_{z}\right)=\zeta v_{F}^{\mathrm{MCT}} \sqrt{2 e \hbar B\left(n-\frac{1}{2}+\frac{\sigma}{4}\right)+\hbar^{2} k_{z}^{2}},
$$

where $k_{z}$ is the momentum along the direction of the applied magnetic field (z-axis) and $\sigma= \pm 1$ describes the splitting due to spin. Let us note that this LL spectrum (of so-called Kane fermions) differs from that of 3D Dirac or Weyl particles, for which the flat $(\zeta=0)$ band does not exist and the

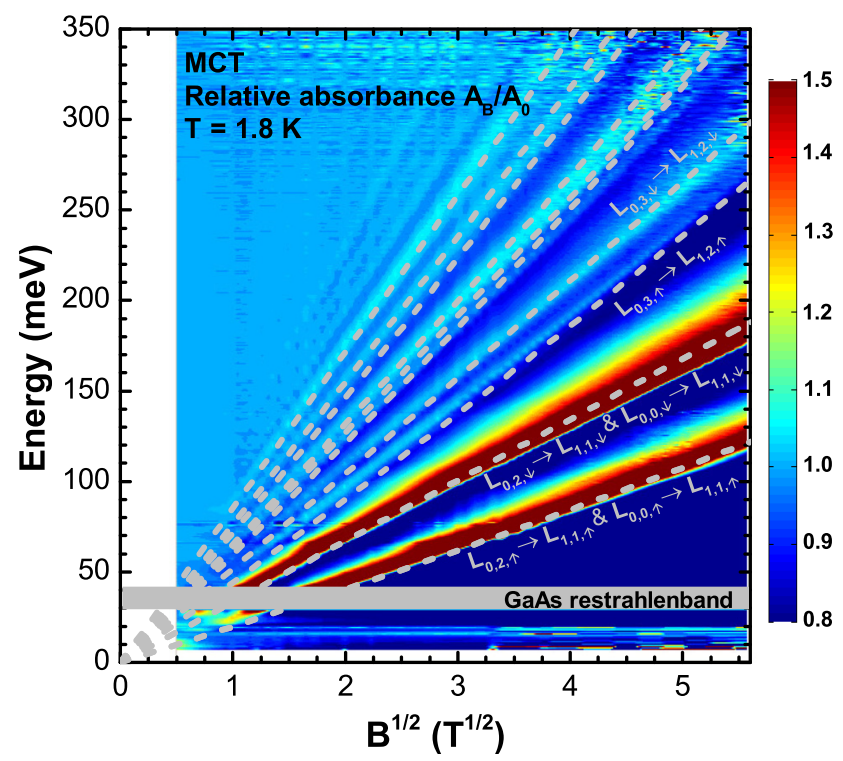

FIG. 3. The relative change of absorbance in the gapless MCT, $A_{B} / A_{B=0}$, plotted as a false colour-map. All the observed resonances clearly follow $\sqrt{B}$ dependence. The dashed lines are calculated positions of inter-LL resonances at $k_{z}=0$ using parameters $v_{F}^{\mathrm{MCT}}=1.06 \times 10^{6} \mathrm{~m} / \mathrm{s}$ and $\Delta=1 \mathrm{eV}$. The presence of the spin-orbit split band, expressed by parameter $\Delta$, does not qualitatively change the LL spectrum, but introduces a weak electronhole asymmetry. 
spin splitting may be formally described by $\sigma= \pm 2$. This implies, for Dirac and Weyl fermions, a doubly spindegenerate LL spectrum: $E_{\zeta, n, \sigma}\left(k_{z}\right)=\zeta v_{F} \sqrt{2 e \hbar B n+\hbar^{2} k_{z}^{2}}$, see, e.g., Ref. 34.

The dispersive character of LLs in MCT, typical also for all other 3D systems, is connected with the particles' motion along the direction of the applied magnetic field. This motion is, as expected, not quantized, but it is not fully decoupled from the cyclotron motion (perpendicular to the field) as is the case of conventional materials with 3D parabolic bands. Instead, the $k_{z}$-dispersion of each LL still depends on the level index $n$. It is also interesting to note that the density of states related to a given LL does not vanish when $k_{z} \rightarrow \infty$ which is the case of conventional 1D systems with parabolic bands. It approaches $\left(\hbar v_{F}\right)^{-1}$, which corresponds to a typical density of states in a 1D Dirac-type channel.

The peculiar spin-splitting in MCT, described by $\sigma= \pm 1$, is fully determined by the Fermi velocity, $v_{F}^{\mathrm{MCT}}$, the only scaling parameter in the LL spectrum of MCT. This surprising fact is another signature of the relativistic-like character of gapless MCT-for truly relativistic particles one cannot separate spin degree of freedom from the orbital motion. Notably, this spin splitting follows a $\sqrt{B}$ dependence, in contrast to conventional materials and also graphene, where the standard Zeeman term implies a linear in $B$ spin splitting, the magnitude of which is determined by the effective $g$-factor ( $g \approx 2$ in graphene with rather weak spinorbit coupling).

In graphene as well as in MCT, the dipole-active interLL excitations follow simple selection rules: $n \rightarrow n+1$ and $n \rightarrow n-1$, active in the right and left circular polarization of the absorbed radiation, respectively. This allows us to unambiguously identify all observed excitations, as shown in Figs. 2 and 3, and to deduce, for instance, the corresponding Fermi velocities: $v_{F}^{\mathrm{G}}=(1.02 \pm 0.01) \times 10^{6} \mathrm{~m} / \mathrm{s}$ and $v_{F}^{\mathrm{MCT}}=(1.06 \pm 0.03) \times 10^{6} \mathrm{~m} / \mathrm{s}$. Surprisingly, we obtain nearly the same Fermi velocity in those two distinctively different systems.

The two most pronounced resonances observed in MCT correspond to excitations from (nearly) degenerate LLs within the flat $(\zeta=0)$ band to the spin-split $n=1 \mathrm{LL}(\zeta=1)$, see Fig. 3 and in the inset of Fig. 4(b). Hence, the energy difference between these two resonances corresponds to the spin-splitting of the $n=1 \mathrm{LL}$, visualizing directly its $\sqrt{B}$ dependence- the well-established signature of ultrarelativistic particles. Notably, such a spin-splitting, when described conventionally, implies an effective $g$-factor, which depends on magnetic field as $B^{-1 / 2}$, and therefore, diverges when $B \rightarrow 0$.

Another distinctive difference in the magneto-optical response of MEG and MCT is the particular shape of inter-LL resonances. In 2D graphene, with discrete LLs, the absorption lines have a symmetric, nearly Lorentzian shape, see, e.g., the $\mathrm{L}_{-1(0)} \rightarrow \mathrm{L}_{0(1)}$ transition Fig. 4(a). The inter-LL resonances in 3D MCT are characterized by an abrupt cutoff on the lowenergy side (due to singularity at $k_{z}=0$ ) and a pronounced shoulder on the high-energy side, as clearly shown in Fig. 4(b). The asymmetric lineshape is a straightforward consequence of the dispersive character of LLs in 3D systems.

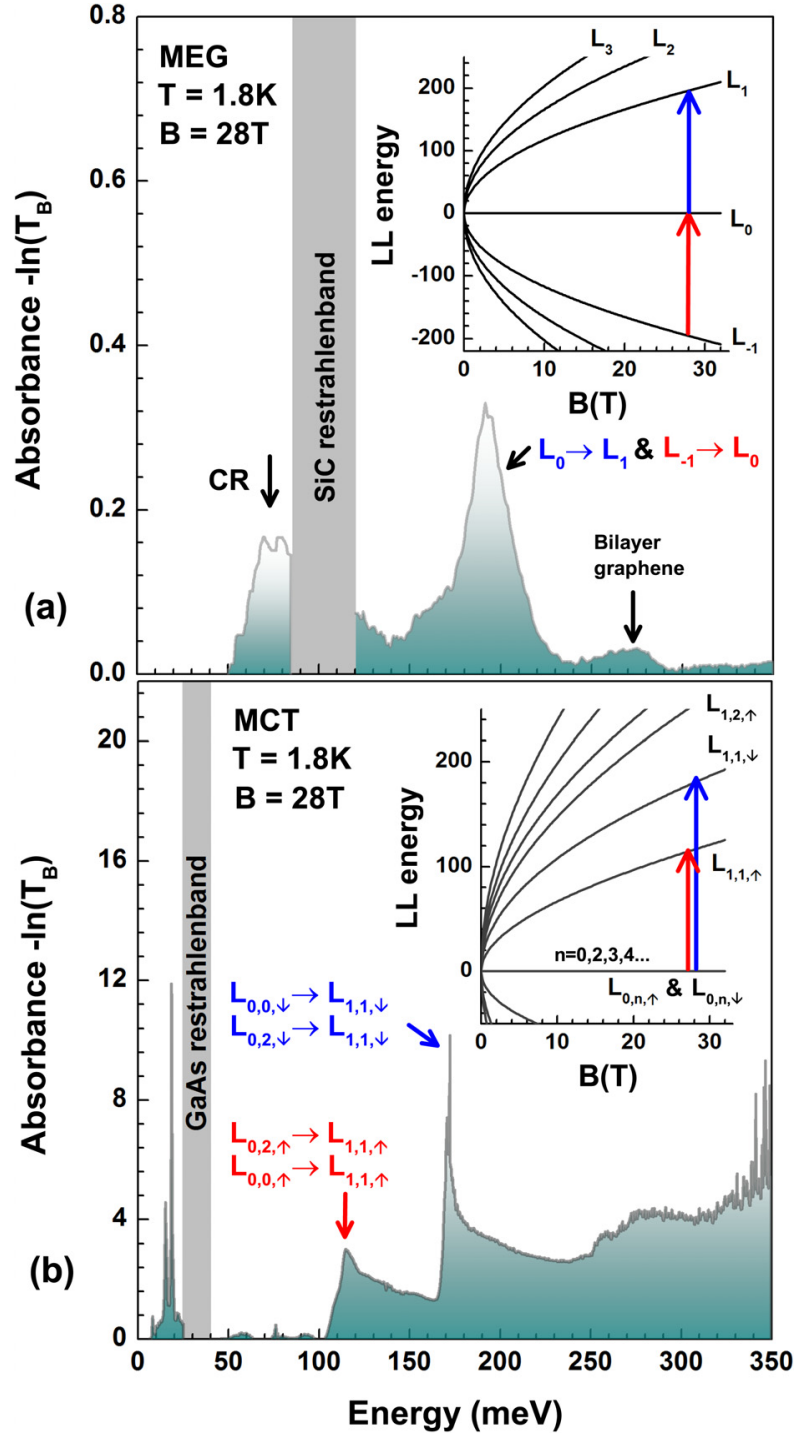

FIG. 4. Absorbance spectra of multilayer epitaxial graphene (plotted for $\hbar \omega>50 \mathrm{meV}$ ) and of the gapless MCT at $B=28 \mathrm{~T}$ in parts (a) and (b), respectively. While MEG exhibits fairly symmetric resonances, see the $\mathrm{L}_{-1(0)} \rightarrow \mathrm{L}_{O(1)}$ transition in the part (a), the 3D character of MCT implies a pronounced high energy tail for each resonance, which is related to the dispersion of particles along the magnetic field direction. The observed resonances are shown in the insets by vertical arrows in the corresponding LL fan chart. The broad line at low energies in the absorbance of MEG is quasi-classical cyclotron resonance absorption in highly doped graphene sheets, which follows a linear in $B$ dependence. ${ }^{35-37}$ In MEG spectra at higher energies, a weak contribution from bilayer graphene inclusions appears. ${ }^{33}$

Interestingly, and in contrast to the conventional 3D systems with parabolic bands, this high-energy tail should be present also for intraband (cyclotron) resonance 3D massless fermions. This is due to the LL dispersion in the direction of the magnetic field, which differs (it depends on the LL index $n$ ) even for levels within one (non-parabolic) band.

\section{CONCLUSIONS}

Concluding, two selected Dirac-type materials-gapless $\mathrm{HgCdTe}$ and multilayer epitaxial graphene-have been probed using infrared magneto-spectroscopy. The presence of massless fermions in both materials implies important 
similarities in the observed magneto-optical responses-in particular, the characteristic $\sqrt{B}$ dependence of all observed resonances. We also find a surprising similarity in the Fermi velocities of charge massless carriers in both materials. However, a closer inspection reveals also differences, which originate in the dimensionality of the studied systems as well as in the particular strength of the spin-orbit interaction. Spin-related effects are rather absent in the optical response of graphene, which exhibits a weak spin-orbit coupling. Instead, we observe a pronounced spin-splitting of levels in $\mathrm{HgCdTe}$ (with a particularly strong spin-orbit interaction) which follows the $\sqrt{B}$ dependence-a well-established signature of relativistic particles.

\section{ACKNOWLEDGMENTS}

The work has been supported by the ERC project MOMB, by EuroMagNET II under the EU Contract No. 228043. This work was supported by the CNRS through LIA TeraMIR project and by DFG under the contract NE 1900/2-1.

${ }^{1}$ J.-C. Charlier, X. Blase, and S. Roche, Rev. Mod. Phys. 79, 677 (2007).

${ }^{2}$ K. S. Novoselov, A. K. Geim, S. V. Morozov, D. Jiang, M. I. Katsnelson, I. V. Grigorieva, S. V. Dubonos, and A. A. Firsov, Nature 438, 197 (2005).

${ }^{3}$ Y. B. Zhang, Y. W. Tan, H. L. Stormer, and P. Kim, Nature 438, 201 (2005).

${ }^{4}$ B. A. Bernevig, T. L. Hughes, and S.-C. Zhang, Science 314, 1757 (2006).

${ }^{5}$ M. König, S. Wiedmann, C. Brüne, A. Roth, H. Buhmann, L. W. Molenkamp, X.-L. Qi, and S.-C. Zhang, Science 318, 766 (2007).

${ }^{6}$ H. Zhang, C.-X. Liu, X.-L. Qi, X. Dai, Z. Fang, and S.-C. Zhang, Nat. Phys. 5, 438 (2009).

${ }^{7}$ M. Z. Hasan and C. L. Kane, Rev. Mod. Phys. 82, 3045 (2010).

${ }^{8}$ X.-L. Qi and S.-C. Zhang, Rev. Mod. Phys. 83, 1057 (2011).

${ }^{9}$ K. Ishizaka, M. Bahramy, H. Murakawa, M. Sakano, T. Shimojima, T. Sonobe, K. Koizumi, S. Shin, H. Miyahara, A. Kimura et al., Nat. Mater. 10, 521 (2011)

${ }^{10}$ Z. K. Liu, B. Zhou, Y. Zhang, Z. J. Wang, H. M. Weng, D. Prabhakaran, S.-K. Mo, Z. X. Shen, Z. Fang, X. Dai et al., Science 343, 864 (2014).

${ }^{11}$ Z. Liu, J. Jiang, B. Zhou, Z. Wang, Y. Zhang, H. Weng, D. Prabhakaran, S. Mo, H. Peng, P. Dudin et al., Nat. Mater. 13, 677-681 (2014).

${ }^{12}$ S. Jeon, B. B. Zhou, A. Gyenis, B. E. Feldman, I. Kimchi, A. C. Potter, Q. D. G. end Robert, J. Cava, A. Vishwanath, and A. Yazdani, Nat. Mater. 13, 851 (2014).

${ }^{13}$ S. Borisenko, Q. Gibson, D. Evtushinsky, V. Zabolotnyy, B. Büchner, and R. J. Cava, Phys. Rev. Lett. 113, 027603 (2014).

${ }^{14}$ Z.-G. Chen, Z. Shi, W. Yang, X. Lu, Y. Lai, H. Yan, F. Wang, G. Zhang, and Z. Li, Nat. Commun. 5, 4461 (2014).

${ }^{15}$ M. Orlita, D. Basko, M. Zholudev, F. Teppe, W. Knap, V. Gavrilenko, N. Mikhailov, S. Dvoretskii, P. Neugebauer, C. Faugeras et al., Nat. Phys. 10, 233 (2014).
${ }^{16}$ M. L. Sadowski, G. Martinez, M. Potemski, C. Berger, and W. A. de Heer, Phys. Rev. Lett. 97, 266405 (2006).

${ }^{17}$ Z. Jiang, E. A. Henriksen, L. C. Tung, Y.-J. Wang, M. E. Schwartz, M. Y. Han, P. Kim, and H. L. Stormer, Phys. Rev. Lett. 98, 197403 (2007).

${ }^{18}$ M. L. Sadowski, G. Martinez, M. Potemski, C. Berger, and W. A. de Heer, Int. J. Mod. Phys. B 21, 1145 (2007).

${ }^{19}$ R. S. Deacon, K.-C. Chuang, R. J. Nicholas, K. S. Novoselov, and A. K. Geim, Phys. Rev. B 76, 081406R (2007).

${ }^{20}$ M. Orlita, C. Faugeras, P. Plochocka, P. Neugebauer, G. Martinez, D. K. Maude, A.-L. Barra, M. Sprinkle, C. Berger, W. A. de Heer et al., Phys. Rev. Lett. 101, 267601 (2008).

${ }^{21}$ M. Orlita, C. Faugeras, J. M. Schneider, G. Martinez, D. K. Maude, and M. Potemski, Phys. Rev. Lett. 102, 166401 (2009).

${ }^{22}$ E. A. Henriksen, P. Cadden-Zimansky, Z. Jiang, Z. Q. Li, L.-C. Tung, M. E. Schwartz, M. Takita, Y.-J. Wang, P. Kim, and H. L. Stormer, Phys. Rev. Lett. 104, 067404 (2010).

${ }^{23}$ M. Orlita, C. Faugeras, R. Grill, A. Wysmolek, W. Strupinski, C. Berger, W. A. de Heer, G. Martinez, and M. Potemski, Phys. Rev. Lett. 107, 216603 (2011).

${ }^{24}$ I. Crassee, J. Levallois, D. van der Marel, A. L. Walter, T. Seyller, and A. B. Kuzmenko, Phys. Rev. B 84, 035103 (2011).

${ }^{25}$ M. Orlita, L. Z. Tan, M. Potemski, M. Sprinkle, C. Berger, W. A. de Heer, S. G. Louie, and G. Martinez, Phys. Rev. Lett. 108, 247401 (2012).

${ }^{26}$ L. Demkó, G. A. H. Schober, V. Kocsis, M. S. Bahramy, H. Murakawa, J. S. Lee, I. Kézsmárki, R. Arita, N. Nagaosa, and Y. Tokura, Phys. Rev. Lett. 109, 167401 (2012).

${ }^{27}$ S. Bordács, J. G. Checkelsky, H. Murakawa, H. Y. Hwang, and Y. Tokura, Phys. Rev. Lett. 111, 166403 (2013).

${ }^{28}$ C. Berger, Z. Song, T. Li, X. Li, A. Y. Ogbazghi, R. Feng, Z. Dai, A. N. Marchenkov, E. H. Conrad, P. N. First et al., J. Phys. Chem. B 108, 19912 (2004).

${ }^{29}$ J. Hass, F. Varchon, J. E. Millán-Otoya, M. Sprinkle, N. Sharma, W. A. de Heer, C. Berger, P. N. First, L. Magaud, and E. H. Conrad, Phys. Rev. Lett. 100, 125504 (2008).

${ }^{30}$ A. C. Ferrari, J. C. Meyer, V. Scardaci, C. Casiraghi, M. Lazzeri, F. Mauri, S. Piscanec, D. Jiang, K. S. Novoselov, S. Roth et al., Phys. Rev. Lett. 97, 187401 (2006).

${ }^{31}$ C. Faugeras, A. Nerriere, M. Potemski, A. Mahmood, E. Dujardin, C. Berger, and W. A. de Heer, Appl. Phys. Lett. 92, 011914 (2008).

${ }^{32}$ Y.-M. Lin, C. Dimitrakopoulos, D. B. Farmer, S.-J. Han, Y. Wu, W. Zhu, D. K. Gaskill, J. L. Tedesco, R. L. Myers-Ward, C. R. Eddy et al., Appl. Phys. Lett. 97, 112107 (2010).

${ }^{33}$ M. Orlita, C. Faugeras, J. Borysiuk, J. M. Baranowski, W. Strupiński, M. Sprinkle, C. Berger, W. A. de Heer, D. M. Basko, G. Martinez et al., Phys. Rev. B 83, 125302 (2011)

${ }^{34}$ P. E. C. Ashby and J. P. Carbotte, Phys. Rev. B 87, 245131 (2013).

${ }^{35}$ A. M. Witowski, M. Orlita, R. Steppniewski, A. Wysmołek, J. M. Baranowski, W. Strupiński, C. Faugeras, G. Martinez, and M. Potemski, Phys. Rev. B 82, 165305 (2010).

${ }^{36}$ I. Crassee, J. Levallois, A. L. Walter, M. Ostler, A. Bostwick, E. Rotenberg, T. Seyller, D. Van Der Marel, and A. B. Kuzmenko, Nat. Phys. 7, 48 (2011).

${ }^{37}$ M. Orlita, I. Crassee, C. Faugeras, A. B. Kuzmenko, F. Fromm, M. Ostler, T. Seyller, G. Martinez, M. Polini, and M. Potemski, New J. Phys. 14, 095008 (2012). 